\title{
MORFOMETRÍA DE LA ESPECIE INVASORA CLARIAS GARIEPINUS (PECES: ACTINOPTERYGII) EN LA LAGUNA LEONERO, GRANMA, CUBA
}

\author{
José Enrique Pérez-Osoria ${ }^{1}$ y Luz Margarita Figueredo Cardona ${ }^{2}$
}

\author{
${ }^{1}$ Empresa Nacional para la Protección de la Flora y la Fauna, Territorio Granma, Carretera de \\ Manzanillo, La Pedrera, Bayamo, Granma, Cuba. ffgranma@enet.cu \\ ${ }^{2}$ Centro Oriental de Ecosistemas y Biodiversidad (BIOECO). José A. Saco No. 601, Esq. Barnada, \\ Santiago de Cuba, 90100, Cuba. luz@bioeco.ciges.inf.cu
}

\section{RESUMEN}

Se evaluó la morfometría del pez gato Clarias gariepinus (Burchell, 1822), especie introducida en Cuba. El estudio se hizo en la laguna Leonero, del municipio Río Cauto, en la provincia Granma. Se capturaron 225 individuos mediante redes, con una luz de malla de 60 a $110 \mathrm{~mm}$, durante marzo, julio y diciembre de 2010. A cada individuo se le tomó: largo total en centímetros, peso total en kilogramos, sexo y peso de las gónadas en gramos. Se determinó el desarrollo gonadal en las hembras. Con los datos obtenidos se calculó el factor de condición y el índice gonadosomático. Del total de individuos muestreados, 149 fueron machos y 76 hembras, para una proporción sexual de $1.96 \mathrm{M} / \mathrm{H}$. El largo total de los peces promedió $61 \mathrm{~cm}$ y el peso $2.0 \mathrm{~kg}$, con una longitud máxima de $140 \mathrm{~cm}$ y un peso máximo de $10.9 \mathrm{~kg}$. No existieron diferencias morfométricas significativas entre sexos. El Factor de Condición demostró el buen estado de salud de la población de peces. En marzo y julio, $53 \%$ de las hembras estaban en los estadios reproductivos III, IV y V, o sea, prevalecieron las fases de maduración más avanzadas.

Palabras clave: Clarias gariepinus, especie invasora, morfometría, laguna Leonero.

Title: Morphometry of invasive species Clarias gariepinus (Fishes: Actinopterygii) in Leonero lagoon, Granma, Cuba.

\section{ABSTRACT}

The morphometry of the introduced catfish Clarias gariepinus (Burchell, 1822) in Leonero lagoon was evaluated, in the Rio Cauto municipality, Granma Province, Cuba. A total of 225 individuals were captured using nets with a grid entrance of 60 to $110 \mathrm{~mm}$, during March, July and December of 2010. The total length in centimeters, total weight in kilograms, sex and gonads weigh was recorded for each individual. We also determined gonad development in females. The condition factors were calculated using this data, as well as the gonadosomatic index. The sample size was 149 males and 76 females, the sexual proportion was $1.96 \mathrm{M} / \mathrm{F}$. Total length of the sample average was $61 \mathrm{~cm}$ and body weight of $2.0 \mathrm{~kg}$. The maximum size founded was $140 \mathrm{~cm}$ and maximum weight of $10.9 \mathrm{~kg}$. No sexual differences between sexes were detected. Condition Factor represented a good health state of the population of fishes. 53 $\%$ of females were in reproductive stages III, IV and V, in March and July.

Keywords: Clarias gariepinus, invasive species, morphometry, Leonero lagoon.

\section{INTRODUCCIÓN}

La especie Clarias gariepinus (Burchell, 1822), conocido como pez gato africano, pertenece a la Familia Clariidae, Orden Siluriformes de la Clase Actinopterygii. La especie tiene su origen en los ríos Níger y Nilo en África; se extiende hacia el sureste de África, en los sistemas ribereños 
de Limpopo, Orange-Vaal, Okavango y Cunene (IGFA, 2001). Su presencia se ha reportado en Levant, Israel, Jordan, Líbano y Siria, además de estar ampliamente introducido en otras partes de África, Europa, Asia y de comercio restringido en Alemania (De Moor y Bruton, 1988). Las especies de Clarias habitan en aguas calmadas desde lagos, arroyos, ríos, pantanos hasta llanuras inundadas sujetas a sequías temporales (Bruton, 1979).

En África, se efectuaron numerosas investigaciones sobre la morfometría de C. gariepinus, como las publicadas por Marshall (1990), en diferentes ríos de Sudáfrica, Burkina Faso, Etiopía, Zambia, Malawi, Botswana, Egipto y Zimbawe. En el mismo continente, también han estudiado su talla Teugels (1986), Rinne y Wanjala (1983) quienes reportaron un peso máximo de $60.0 \mathrm{~kg}$ y Van Oijen (1995) encontraron individuos de hasta $90.0 \mathrm{~cm}$.

En Cuba, se han realizado escasos estudios sobre de C. gariepinus que analizan talla y peso, como los efectuados en Ciénaga de Zapata (CITMA Matanzas, 2005), donde registraron ejemplares con pesos predominantes entre 1.5 y $2.0 \mathrm{~kg}$. Investigaciones similares realizaron Pérez et al. (2005) en el río Guareira, que incluyeron el análisis del estadio gonadal. Los autores obtuvieron promedios de peso y longitud de $4.2 \mathrm{~kg}$ y $79.6 \mathrm{~cm}$, respectivamente.

En 1999, se realizó en Cuba la primera introducción de Clarias gariepinus, con fines de investigación científica, desde Malasia. La segunda introducción se produjo en el 2000 junto con C. macrocefalus, desde Tailandia, con la finalidad de cruzar ambas especies y producir larvas híbridas destinadas a los cultivos intensivos en estaques de alevinaje de todo el país (MIP, 2007). Las dos especies son dañinas para la fauna autóctona, debido a su capacidad para devorar huevos y alevines, por lo que constituyen una gran amenaza para las poblaciones piscícolas, tanto nativas como comerciales, pues pueden provocar cambios en la estructura trófica, el desplazamiento de las especies nativas y la posible transmisión de enfermedades (Gutiérrez, 2006).

Cuba tiene regulaciones que restringen el uso de C. gariepinus solo a los cultivos intensivos, que se hacen en estaciones de alevinaje y granjas de ceba (MINAL, 2009). Existen tres vías de introducción de Clarias a la laguna Leonero: a través de los embarques de siembra de tilapia; por el sistema de canales conectados con la presa Cauto El Paso, dedicada al suministro de agua para el cultivo del arroz; y la interacción hidrográfica que se produce de manera natural en las diferentes bifurcaciones del río Cauto y arroyos que tributan al área, como el Jobabo.

\section{OBJETIVO}

- Evaluar la morfometría de la población adulta de C. gariepinus en la laguna Leonero, perteneciente al municipio Río Cauto, provincia Granma.

\section{MATERIALES Y MÉTODOS}

La laguna Leonero es un acuatorio seminatural con una extensión de 5, 448 ha y 3, 500 ha de espejo de agua. Se encuentra ubicada al noreste del Refugio de Fauna Delta del Cauto, provincia Granma ( $77^{\circ} 4 ’ 39,83$ ” W, 20³9' 55,99” N). El área ocupa una parte considerable de la desembocadura del río Cauto, el mayor ecosistema deltaico de Cuba y el Caribe Insular.

Durante marzo, julio y diciembre de 2010, se realizaron muestreos biológico-pesqueros. Se capturaron individuos de C. gariepinus, mediante redes agalleras con una luz de malla de 60 a $110 \mathrm{~mm}$. A cada ejemplar se le determinó el sexo y se le midió la talla con una cinta métrica de $1 \mathrm{~cm}$ de precisión, peso total con una pesola de $5 \mathrm{~kg}( \pm 0.1 \mathrm{~g})$ y estado general de desarrollo de los individuos (Factor de Condición). El peso de las gónadas se determinó con una balanza analítica PRECISA 180A ( $\pm 0.1 \mathrm{mg})$ y se estableció el grado de madurez sexual en las hembras. 
Se determinó la estructura de la población por clase de talla, con intervalos de $10 \mathrm{~mm}$ y la proporción M:H.

Con los datos de peso y longitud de cada individuo se calculó el factor de condición o robustez para determinar el estado general de desarrollo de los individuos capturados (Fulton, 1902). La fórmula utilizada fue la siguiente:

$$
\mathrm{FC}=\mathrm{PT} \times 100 / \mathrm{L}^{3}
$$

donde:

$$
\begin{aligned}
& \mathrm{FC}=\text { Factor de Condición } \\
& \mathrm{PT}=\text { Peso Total } \\
& \mathrm{L}=\text { Longitud total }
\end{aligned}
$$

Se consideraron individuos adultos los ejemplares con tallas entre 32 y $140 \mathrm{~cm}$, según los Procedimientos Operacionales de Trabajo en Cuba (MINAL, 2009). La madurez sexual se determinó mediante la observación de las características macroscópicas externas y de la coloración de las gónadas, según la escala establecida en los Procedimientos Operacionales de Trabajo del Ministerio de la Industria Pesquera de Cuba, actualmente Ministerio de la Industria Alimentaria (MIP, 2003). Esta escala presenta modificaciones a las descritas por Owiti y Dadzie (1989) y De Graaf y Janssen (1996).

Las descripciones de cada estadio aparecen a continuación:

Estadio I (Virgen): Ovario incoloro a carmelita traslúcido, lanceolado y en apariencia lobular, ocupando la cuarta parte posterior de la cavidad del cuerpo.

Estadio II (Virgen en desarrollo): Ovario traslúcido, de color carmelita y ocupando alrededor de la tercera parte de la longitud de la cavidad peritoneal. Los ovocitos individualmente visibles, como manchitas diminutas.

Estadio III (Madurando): Ovario opaco, de color verde-carmelita, ocupando alrededor de la mitad de la longitud de la cavidad abdominal. Huevos visibles como gránulos verdeamarillentos o amarillo-carmelitas y presencia de capilares sanguíneos alrededor del ovario.

Estadio IV (Maduro): Ovario alargado, opaco y de color verde-carmelita. Huevos llenos de yemas (vitelo) y claramente visibles. El ovario ocupa la cuarta quinta parte de la cavidad peritoneal. Presencia de una red capilar altamente desarrollada. Los huevos salen libremente con presión sobre el vientre.

Estadio V (Desove): Huevos traslúcidos y aplanados, visibles como una corona esférica carmelita rojiza.

Se calculó el índice gonadosomático (Ferrer, 1988), para determinar madurez de las gónadas y posible época de desove de la especie en la laguna. Este se obtuvo con la fórmula:

$$
\mathrm{IG}=\mathrm{PG} / \mathrm{PT} \times 100
$$

donde:

$$
\begin{aligned}
& \mathrm{IG}=\text { Índice gonadosomático } \\
& \text { PG }=\text { Peso de las gónadas } \\
& \text { PT }=\text { Peso total del individuo }
\end{aligned}
$$


Se aplicó la prueba de normalidad de Kolmogorov Smirnov (Massey, 1951) con el programa STATISTICA 7.0, Statsoft 7.0, 2004 y como los datos no fueron normales se aplicó estadística no paramétrica. Como estadísticos descriptivos se determinaron la media, error estándar, coeficiente de variación, la mediana, la moda y la frecuencia modal. Se estimaron los intervalos de confianza de las proporciones sexuales con la distribución $\mathrm{F}$ según el método de Clopper y Pearson (1934). Las medidas morfométricas entre sexos se compararon por una prueba $U$ de Mann Whitney. Los rangos de talla se seleccionaron aplicando el cálculo de cuartiles y percentiles en sus histogramas respectivos, usando el programa STATISTICA 7.0, Statsoft 7.0, 2004. Todas las pruebas estadísticas se hicieron de una cola y con un nivel de significación del $5 \%$.

\section{RESULTADOS}

Se capturaron 225 ejemplares de C. gariepinus: 149 machos (66\%, IC: 0.502- 0.664) y 76 hembras $(34 \%$, IC: 0.227 - 0.377). La proporción poblacional entre sexos fue de 1.96:1 $(\mathrm{M}: \mathrm{H})$. También se encontraron más machos que hembras, con los mayores en julio y diciembre (Tabla 1).

Tabla 1. Proporción sexual en las capturas de C. gariepinus por cada mes de muestreo en la Laguna Leonero, Cuba.

\begin{tabular}{lcccc}
\hline Fecha de muestreo & Hembras $(\mathrm{H})$ & Machos $(\mathrm{M})$ & Total & Proporción sexual $(\mathrm{M}: \mathrm{H})$ \\
\hline Marzo & 20 & 28 & 48 & $1.40: 1$ \\
Julio & 24 & 47 & 71 & $1.96: 1$ \\
Diciembre & 32 & 74 & 106 & $2.31: 1$ \\
\hline \multicolumn{1}{c}{ Total } & 76 & 149 & 225 & $1.96: 1$ \\
\hline
\end{tabular}

La longitud total promedio de los especímenes fue de $61 \mathrm{~cm}$ y el peso total promedio de 2.0 $\mathrm{kg}$ (Tabla 2). Los resultados de la talla promedio fueron casi similares para hembras y machos, igualmente ocurrió con el peso, reportándose para las hembras un peso promedio de $2.2 \mathrm{~kg}$ y para los machos $2.0 \mathrm{~kg}$. La talla máxima encontrada fue de $140 \mathrm{~cm}$ y la mínima de $31 \mathrm{~cm}$. En cuanto al peso, el máximo fue de $10.9 \mathrm{~kg}$ y el mínimo $0.5 \mathrm{~kg}$. No existieron diferencias significativas entre las variables morfométricas para hembras y machos, por lo que se trabajó en el análisis de los datos con los valores de la muestra total.

La mediana en el largo total dio un valor de $60 \mathrm{~cm}$ y el peso de $2.1 \mathrm{~kg}$; valores bastante similares aportó la moda para ambas variables morfométricas, según la distribución de frecuencia.

Tabla 2. Estadísticos descriptivos de las variables morfométricas en la población de C. gariepinus en la laguna Leonero, Granma.

\begin{tabular}{|c|c|c|c|c|c|c|}
\hline Variable & Media & $\begin{array}{l}\text { Error } \\
\text { estándar }\end{array}$ & Mínimo & Máximo & $\begin{array}{l}\text { Coef. } \\
\text { Var. }\end{array}$ & $\begin{array}{c}\text { U de Mann } \\
\text { Whitney (p) }\end{array}$ \\
\hline \multicolumn{7}{|l|}{ Hembras $(n=76)$} \\
\hline Largo total $(\mathrm{Lt})(\mathrm{cm})$ & 61.41 & 0.766 & 31.0 & 140.0 & 18.73 & \multirow{4}{*}{$\begin{array}{l}\text { Lt: } 0.265(\mathrm{p}=0.79) \\
\text { Pt: } 0.855(\mathrm{p}=0.39)\end{array}$} \\
\hline Peso total $(\mathrm{Pt})(\mathrm{kg})$ & 2.037 & 0.078 & 0.50 & 10.90 & 57.56 & \\
\hline Factor de Condición (FC) & 1.361 & 0.084 & 0.155 & 11.227 & 92.15 & \\
\hline \multicolumn{6}{|l|}{ Machos (n=149) } & \\
\hline Largo total $(\mathrm{Lt})(\mathrm{cm})$ & 61.72 & 0.986 & 45.5 & 140.0 & 19.50 & \multirow[t]{3}{*}{ FC: $0.483(\mathrm{p}=0.63)$} \\
\hline Peso total $(\mathrm{Pt})(\mathrm{kg})$ & 1.98 & 0.080 & 0.60 & 7.60 & 49.31 & \\
\hline Factor de Condición (FC) & 1.325 & 0.091525 & 0.312 & 10.640 & 84.332 & \\
\hline
\end{tabular}


E1 93\% de la muestra presentó valores entre 45 a $74 \mathrm{~cm}$ y la clase de talla de mayor cantidad de individuos fue de 55 a $65 \mathrm{~cm}$, representando el 45\%. A partir de los $75 \mathrm{~cm}$ se encontró la menor cantidad de ejemplares (Fig. 1). Teniendo en cuenta que $31 \mathrm{~cm}$ no corresponde a individuos adultos, sino juveniles, se discriminaron los datos correspondientes a los dos ejemplares que tenían esta talla.

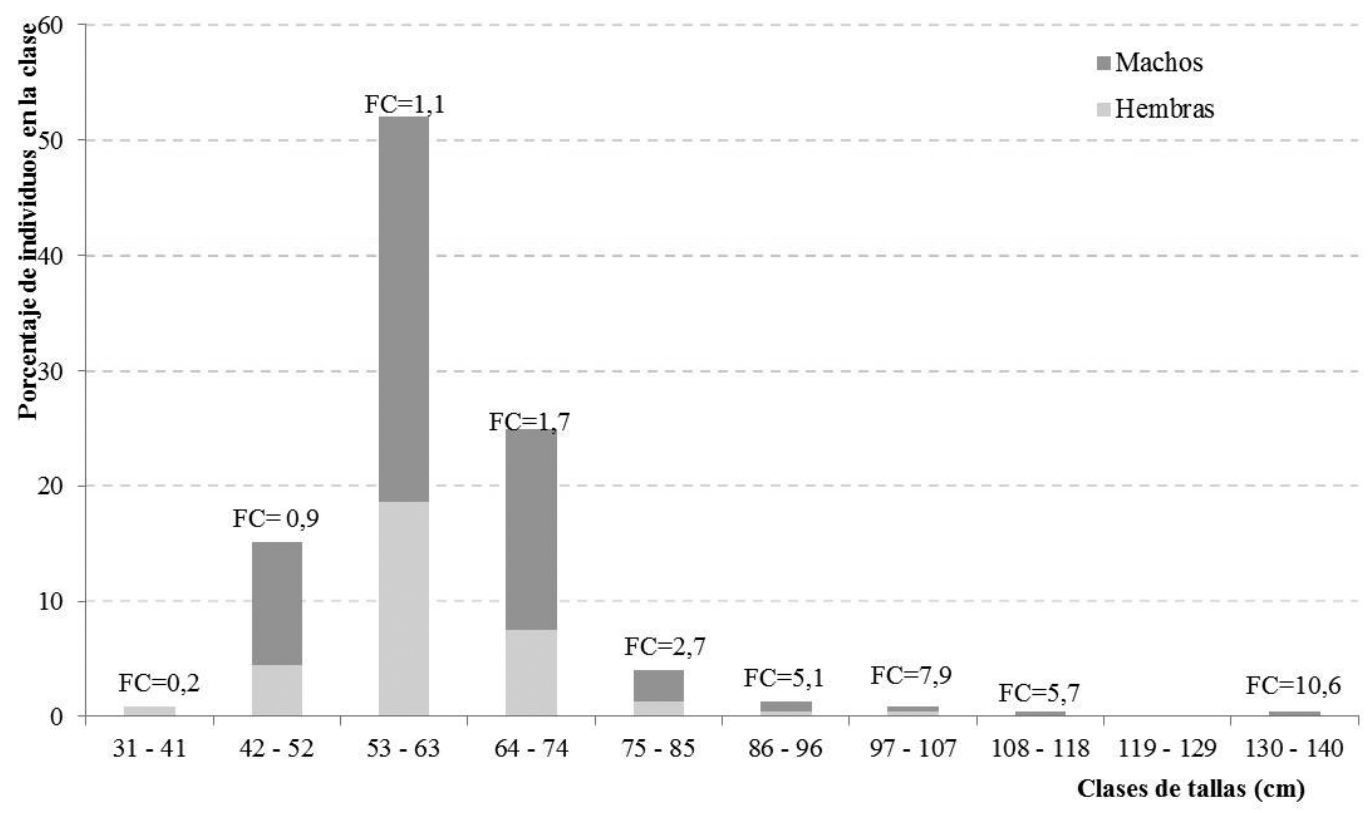

Figura 1. Porcentaje de individuos de C. gariepinus por clase de talla y sexo en la laguna Leonero, Granma, Cuba.

El factor de condición de la muestra dio un valor mayor que 1 (Tabla 2). Solo el 37\% de la misma presentó valores menores que 1 . Del total de las hembras el $67 \%$ (51) tenían un FC mayor o igual que 1 y el $62 \%$ de los machos presentan la misma condición. De manera general, la población estudiada cuenta con buen estado de salud y no existen diferencias significativas entre sexos.

En el examen macroscópico, según las características morfológicas de las gónadas, los individuos se agruparon en los siguientes estadios de madurez sexual, que se diferenciaron significativamente en el peso de las gónadas:

Estadio I (Virgen): 34 hembras con un peso promedio de las gónadas de $1.9 \mathrm{~g}$.

Estadio II (Virgen en desarrollo): dos individuos con un peso promedio gonadal de $7 \mathrm{~g}$.

Estadio III (Madurando): tres hembras con un peso promedio de las gónadas de $11.7 \mathrm{~g}$.

Estadio IV (Maduro): 35 hembras con 82.7 g de peso promedio de las gónadas.

Estadio V (Desove): dos hembras con un peso promedio de las gónadas de $15 \mathrm{~g}$.

Prueba de Kruskal-Wallis: $\mathrm{H}(5, \mathrm{~N}=76)=52.29 \mathrm{p}<0.001$ 


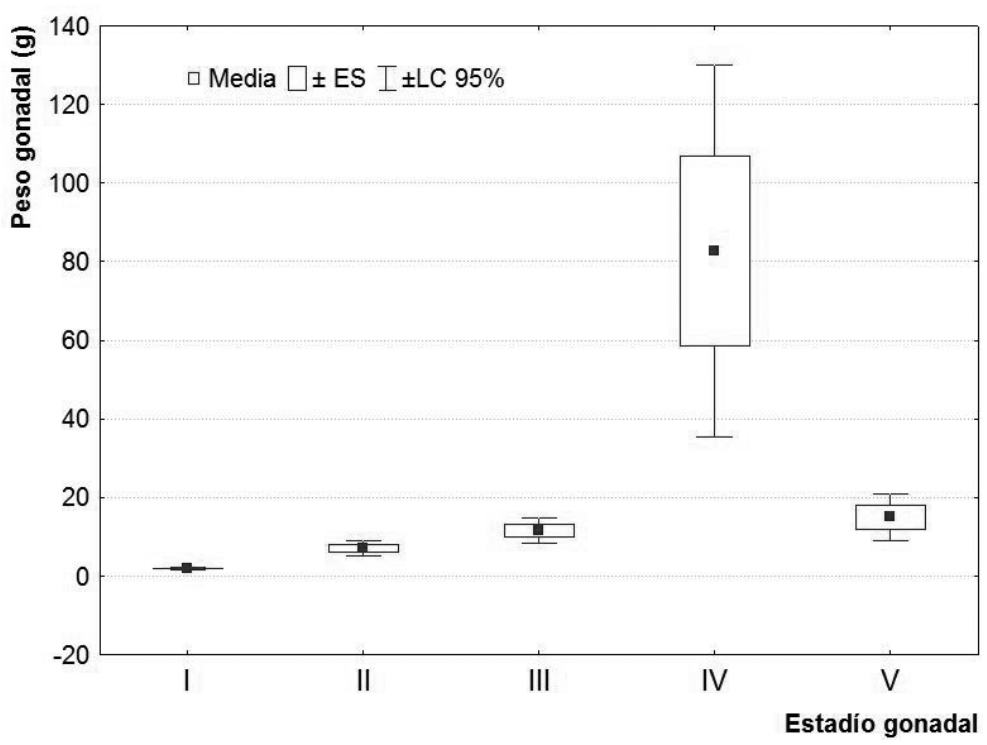

Figura 2. Peso gonadal por estadio gonadal en C. gariepinus en la laguna Leonero, Granma, Cuba.

En la población adulta de C. gariepinus, el 46\% de las hembras examinadas se encontraban en estadio IV y el 44\% en estadio I. Al hacer el análisis por meses resultó, que en marzo y julio predominó el estadio IV con 60 y 95\% respectivamente, no así en noviembre, donde el 100\% de las hembras capturadas se encontraban en estadio I (Fig. 3).

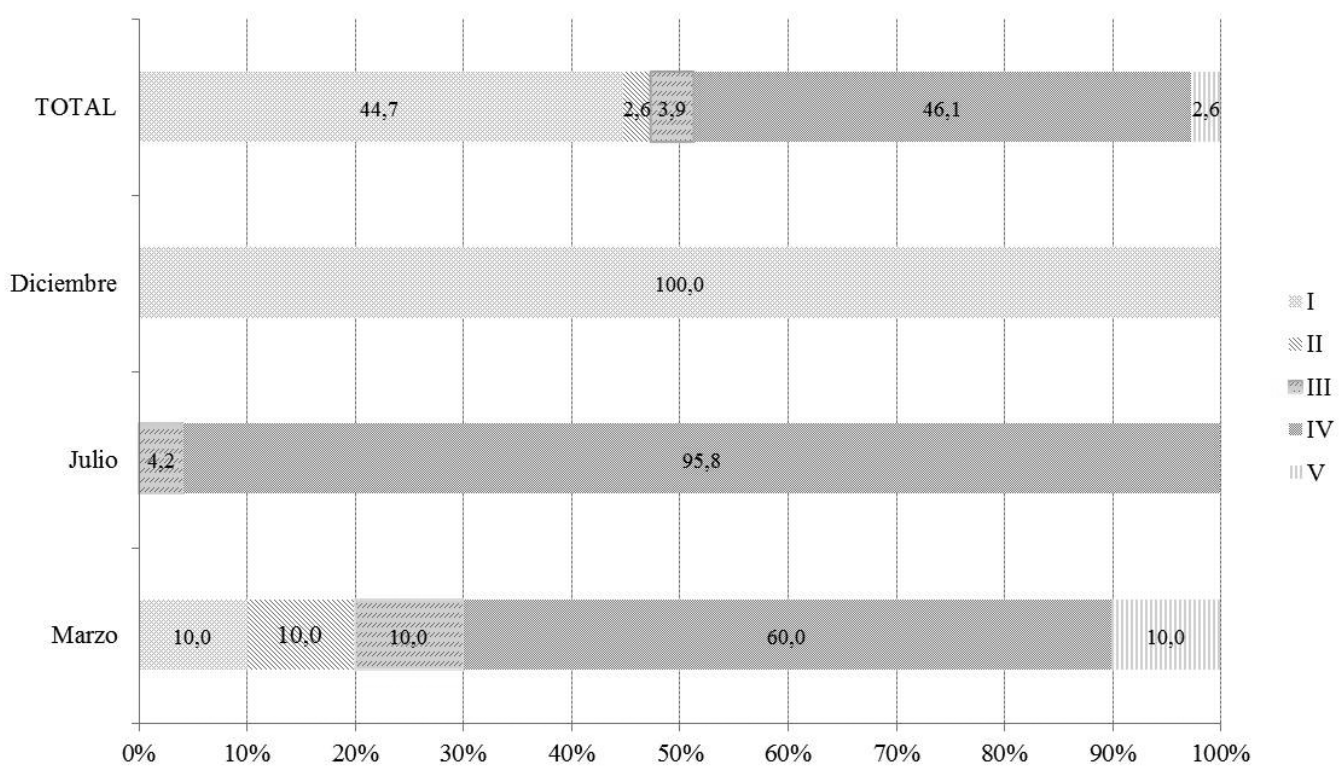

Figura 3. Cantidad de hembras de C. gariepinus por estadio gonadal en cada época de muestreo en la laguna Leonero, Granma, Cuba.

El índice gonadosomático aumenta en la medida que se incrementa la madurez de las gónadas, fue mayor en los estadios IV y $\mathrm{V}$ y menor en el estadio I, II y III. Aunque en la muestra el estadio V no presentó grandes valores siempre fue mayor que los estadios primarios, demostrándose la proporcionalidad del índice. 


\section{DISCUSIÓN}

La proporción sexual encontrada de C. gariepinus, en la laguna Leonero, fue de 1.96M: 1H. Esta prevalencia de machos ocurrió en las tres fechas de muestreo, con mayor énfasis para julio y diciembre. En julio, dentro de la temporada lluviosa, este comportamiento pudiera estar influenciado a que las hembras tienden a migrar buscando sitios menos profundos para el desove. Esto no justifica el balance positivo hacia machos encontrados, pues la disponibilidad de la captura es para ambos sexos. No obstante, puede ser una conducta natural de la especie, lo que coincide con lo encontrado por Pérez et al. (2005), en el río Guareira y sus afluentes en Ciénaga de Zapata (Matanzas).

La prevalencia de machos sobre hembras en los grupos de edades mayores se obtuvo, además, en investigaciones realizadas en los ríos Elands (Van der Waal, 1972), Lower Shire (Willoughby y Tweddle, 1978) y Zambesi (Clay, 1984). También se observó en lagos de Sudáfrica (Bruton y Allanson, 1980; Harrison, 2001) y Kenya (Britton y Harper, 2006).

En la laguna Leonero, el pez de mayor talla fue un macho de $140 \mathrm{~cm}$, superior a lo encontrado en Cuba por Pérez et al. (2005), en el río Guareira de Ciénaga de Zapata. Este constituye el mayor registro publicado para Cuba respecto a la talla de C. gariepinus.

El registro de longitud encontrado en esta investigación es superior a lo documentado en las investigaciones de Yalçin et al. (2001), en el río Asi (Turquía), quienes registraron un espécimen hembra de $82.6 \mathrm{~cm}$ y solo $4.02 \mathrm{~kg}$ de peso. Van Oijen (1995), en el lago Victoria, halló individuos de hasta $90 \mathrm{~cm}$. En el valle Shire, Malawi, el ejemplar de mayor talla encontrado fue un macho de $100 \mathrm{~cm}$ y $8.5 \mathrm{~kg}$ de peso (Willoughby y Tweddlle, 1978). En el lago Sibaya, el pez más largo midió $108.8 \mathrm{~cm}$ y un peso de $8.7 \mathrm{~kg}$ (Bruton y Allanson, 1980). La mayor longitud total registrada a nivel mundial para la especie es de $170 \mathrm{~cm}$, en una base de datos y lo refiere como dudoso (IGFA, 2001).

Los autores anteriormente referidos realizaron sus investigaciones en ecosistemas de donde es oriunda la especie. Sin embargo, en Cuba, C. gariepinus ha encontrado condiciones favorables para su desarrollo, disponibilidad de alimentos y ausencia de controladores biológicos naturales.

Aunque se analizaron ejemplares de hasta $10.9 \mathrm{~kg}$, los resultados de peso promedio en la muestra, $2.1 \mathrm{~kg}$, son inferiores al encontrado por Pérez et al. (2005), $4.3 \mathrm{~kg}$, en el río Guareira de Ciénaga de Zapata. Lo mismo ocurrió con las tallas promedio. En ambas investigaciones, difiere el tamaño muestral que fue mucho menor en el muestreo realizado en el río Guareira. Además fueron distintas las artes de pesca utilizadas; Pérez et al. (2005) usaron nasas, las que son muy efectivas para la captura de esta especie. Sin embargo, en este estudio no se empleó la misma porque tiene una abertura de malla poco selectiva para las tallas que se pretendían obtener. Además, presenta el riesgo de capturar otras especies importantes de la fauna dulceacuícola que no son de interés para esta investigación.

Al comparar los datos obtenidos en esta muestra con lo registrado en las investigaciones realizadas en África, las variables morfométricas de longitud y peso son muy cercanas a las reportadas por Van der Waal (1972, 2000), Willoughby y Tweddle (1978), Bruton y Allanson (1980), Teugels (1986), Marshall (1990), Van Oijen (1995) y Yalçin et al. (2001). Estos autores tampoco encontraron diferencias significativas en las variables morfométricas para hembras y machos.

El hecho de que la mayor cantidad de individuos se encuentre en las tallas entre 45 y 74 $\mathrm{cm}$ se debe a la selectividad de las redes utilizadas, que son efectivas en la captura de estos 
individuos. Además, responde a una clase de edad determinada. En las clases de talla mayores de $75 \mathrm{~cm}$, se agrupó un menor número de ejemplares, lo que constituye un resultado esperado si se tiene en cuenta que este acuatorio está sometido a la pesca intensiva por la Base de Pesca de Leonero (BP Leonero, 2010). A ello se añade que las poblaciones de individuos de mayor longitud tienden a ser menos numerosas en el medio natural.

La clase de talla predominante $(55-64 \mathrm{~cm})$ corresponde a individuos que pudieran tener entre 3 y 5 años de edad, si se consideran los valores del incremento relativo de largo y peso obtenidos por Hogendoorn (1983), Clay (1984), Quick y Bruton (1984), Marshall (1990), Rognon et al. (1998), Yalçin et al. (2001) y Turan et al. (2005) para esta especie, en cuerpos de agua bajo condiciones ecológicas similares a la laguna Leonero. Estos datos permiten estimar la captura efectiva de individuos de talla comercial en el acuatorio.

Los resultados obtenidos del factor de condición, donde el 63\% de la muestra presentaba valores mayores o iguales que 1 demuestran que la población tenía un buen estado de salud. Esto se debe a que los individuos encontrados eran sanos, sin síntomas de parasitismo ni alguna otra enfermedad que amenazara su desarrollo. Además, la especie tiene una buena alimentación debido a la disponibilidad de alimentos en la laguna.

A lo anterior se añade que $C$. gariepinus es un pez omnívoro, oportunista, con amplio espectro en sus hábitos alimentarios, lo que corrobora lo encontrado por De Moor y Bruton (1988), De Graaf y Janssen (1996), Okeyo (1998), Toledo et al. (2001) y Manyla Times (2002). Estos autores obtuvieron cifras del factor de condición similares a las registradas en esta investigación.

En marzo y julio, se encontró la mayor cantidad de hembras en estadio IV y $\mathrm{V}$ y por consiguiente, los mayores valores del IG; coinciden estos meses con la época de lluvia. Lo anterior permite inferir que este periodo podría ser el pico de desove masivo de la especie en la laguna. Ello se corrobora con los propios resultados del muestreo realizado en diciembre donde solo se hallaron hembras en estadio I; además se conoce que este mes es uno de los más secos del año.

El predominio de hembras en estadio gonadal maduro coincide con lo encontrado por Pérez et al. (2005) en el río Guareira (Ciénaga de Zapata), en ambos casos la época de muestreo era la misma (marzo y julio), meses que se corresponden con la fase de maduración (Fig. 3).

Los valores del índice gonadosomático de las hembras en estadio IV y V fueron altos, lo que indica el buen estado de las gónadas y su correspondencia con el peso gonadal. El estadio $\mathrm{V}$ tuvo una ligera tendencia a disminuir el IG, esto pudo estar influenciado por la pérdida de huevos en la captura y el manejo del individuo, debido a que este es el estadio de desove.

\section{CONCLUSIONES}

La población de C. gariepinus de la laguna Leonero, Granma, tiene una proporción sexual favorable a los machos, no presenta diferencias morfométricas significativas entre sexos y tiene un buen estado de salud, mostrado por el factor de condición. Predominan los individuos con las clases de talla entre 55 y $64 \mathrm{~cm}$ que se corresponde con edades entre 3 y 5 años. Las hembras con estadios más avanzados de maduración prevalecen en marzo y julio. 


\section{AGRADECIMIENTOS}

Agradecemos la colaboración recibida por Omar Labrada y los técnicos del Refugio de Fauna Delta del Cauto, quienes nos apoyaron en el proceso de muestreo biológico pesquero; también a los pescadores de la Base de Pesca Leonero por la colaboración en la captura de los individuos.

\section{LITERATURA CITADA}

BP Leonero. 2010. Base de Pesca Leonero. Informe de siembra de alevines de Clarias gariepinus. PESCAGRAM.

Britton, J. R. y D. M. Harper. 2006. Length-weight relationships of fish species in the freshwater rift valley lakes of Kenya. J. Appl. Ichthyol 22: 334-336.

Bruton, M. N. 1979. The fishes of Lake Sibaya. 162-245 pp. En B.R. Allanson, ed. Lake Sibaya. Monographiae Biologicae 36. Dr. W. Junk bv Publishers, Hague.

Bruton, M. N. y B. R. Allanson. 1980. The growth of Clarias gariepinus in Lake Sibaya, South Africa. S. Afr. J. Zool. 15: 7-15.

CITMA Matanzas. 2005. Informe de la Ciénaga de Zapata (Reserva de la biosfera y Sitio Ramsar). Sobre la introducción de Clarias en el territorio. (Inédito).

Clay, D. 1984. Production of the African catfish (Clarias gariepinus). I. Growth, mortality and yield south of the Zambesi. J. Limnol. Soc. S. Afr. 10: 16-24.

Clopper, C. J. y E. S. Pearson. 1934. The use of confidence or fiducial limits illustrated in the case of the binomial. Biometrika 26: 404-413.

De Graaf, G. y J. Janssen. 1996. Handbook on the artificial reproduction and pond rearing of the African catfish Clarias gariepinus in Sub-Saharan Africa FAO Fisheries Technical Paper 362.

De Moor, I. J. y M. N. Bruton. 1988. Atlas of alien and translocated indigenous aquatic animals in southern Africa. A report of the Committee for Nature Conservation Research National Programme for Ecosystem Research. South African Scientific Programmes Report No. 144. 310 p. Port Elizabeth, South Africa.

Ferrer, M. 1988. Madurez sexual, Diámetro de Huevos, Fecundidad y factores relacionados de la lisa (Mugil curema Valenciennes, 1836) del Lago de Maracaibo. Zootecnia Tropical, 6 (1 y 2): 81-112.

Fulton, T. 1902. Rate of growth of sea-fishes.Sci. Invest. Fish. Div. Scot. Rept.1-22.

Gutiérrez, F. 2006. Estado de conocimiento de especies invasoras. Propuesta de lineamientos para el control de los impactos. Instituto de Investigación de Recursos Biológicos Alexander von Humboldt, Bogotá, D.C. Colombia. 156 pp.

Harrison, T. D. 2001. Length-weight relationships of fishes from South African estuaries. J. Appl. Ichthyol. 17(1): 46-48.

Hogendoorn, H. 1983. Growth and production of the African catfish, Clarias lazera (C. y V.). III. Bioenergetic relations of body weight and feeding level. Aquaculture 35: 1-17. 
IGFA. 2001. Database of IGFA angling records until 2001. IGFA, Fort Lauderdale, USA.

Manyla Times. 2002. La venganza de las especies exóticas (Artículo publicado en el periódico Manyla Times de Filipinas). Tuesday, April 23, 2002. Science Faction.

Marshall, B. E. 1990. Growth performance of the African catfish Clarias gariepinus (Clariidae). Fishbyte 8(2): 17-19.

Massey, F. J., Jr. 1951. The Kolmogorov-Smirnov test for goodness of fit. Journal of the American Statistical Association, 46: 68-78.

MINAL (Ministerio de la Industria Alimentaria). 2009. Instrucción Ministerial. Anexo Plan de manejo de la especie Clarias garepinus en cultivos de organismos acuáticos. Ministerio de la Industria Alimentaria.

MIP (Ministerio de la Industria Pesquera). 2003. Procedimiento para evaluar el posible impacto de Clarias gariepinus en embalses. Procedimientos Operacionales de Trabajo.

MIP (Ministerio de la Industria Pesquera). 2007. Información del pez gato africano Clarias gariepinus. Dirección de Pesca y Acuicultura. La Habana. Cuba. 12 pp.

Okeyo, D. O. 1998. Updating names, distribution and ecology of riverine fish of Kenya in the Athi-Galana-Sabaki River drainage system. Naga ICLARM Q. 21(1): 44-53.

Owiti, D. O. y S. Dadzie S. 1989. Maturity, fecundity and the effect of reduced rainfall on the spawning rhythm of a siluroid catfish, Clarias mossambicus (Peters). Aquaculture and fisheries management, 20: 355-368.

Pérez, E., A. Hurtado, E. Abreu, E. Sánchez, I. González, G. Sosa y L. Guerra. 2005. Manejo de las poblaciones de Clarias en los ecosistemas acuáticos del río Guareira y sus afluentes. PESCAMAT. CITMA Matanzas. Parque Nacional Ciénaga de Zapata. Abril, 2005. 10 pp. (Inédito).

Quick, A. J. R. y M. N. Bruton. 1984. Age and growth of Clarias gariepinus (Pisces: Clariidae) in the P.K. le Roux Dam, South Africa. S. Afr. J. Zool. 19(1): 37-45.

Rinne, J. N. y B. Wanjala. 1983. Maturity, fecundity, and breeding season of the major catfishes (suborder: Siluroidea) in Lake Victoria, East Africa. J. Fish Biol. 23: 357-363.

Rognon, X., G. G. Teugels, R. Guyomard, P. Galbuseras, M. Andriamanga, F. Volckaert y J. F. Agnèse. 1998. Morphometric and allozyme variation in the African catfishes Clarias gariepinus and C. anguillaris. J. Fish Biol. 53(1): 192-207.

Teugels, G. G. 1986. A systematic revision of the African species of the genus Clarias (Pisces; Clariidae). Ann. Mus. R. Afr. Centr., Sci. Zool., 247: 199 pp.

Toledo, S. G., J. E. Llanes, J. M. Lazo e I. Fernández. 2001. Aquaculture Feeding. Centro de Preparación Acuícola de Mampostón. Planta Piloto de Investigación-Producción de Alimentos No Convencionales. La Habana. Cuba. (Presentación).

Turan, C., F. Yalçin, E. Turan, N. Okur e I. Akyurt. 2005. Morphometric comparisons of African catfish, Clarias gariepinus, populations in Turkey. Folia Zool. 54(1-2): 165-172. 
Van der Waal, B. C. W. 1972. 'n Ondersoek na aspekte van die ekologie, teelt en produksie van Clarias gariepinus (Burchell, 1822). Rand Afrikaans University, South Africa. M.S. thesis. (in Afrikaans).

Van der Waal, B. C. W. 2000. Fish as a resource in a rural river catchment in the Northern Province, South Africa. Afr. J. Aquat. Sci. 25: 56-70.

Van Oijen, M. J. P. 1995. Appendix I. Key to Lake Victoria fishes other than haplochromine cichlids. p. 209-300. En F. Witte and W.L.T. van Densen, eds. Fish stocks and fisheries of Lake Victoria. A handbook for field observations. Samara Publishing Limited, Dyfed, Great Britain.

Willoughby, W. y D. Tweddle. 1978. The ecology of the catfish, Clarias gariepinus and Clarias ngamensis in the Shire Valley, Malawi. J. Zool. 186(4): 507-534.

Yalçin, S., K. Solak e I. Akyurt. 2001. Certain Reproductive Characteristics of the Catfish (Clarias gariepinus Burchell, 1822) Living in the River Asi, Turkey. Turkish Journal of Zoology 25: 453-460.

[Recibido: 07 de septiembre, 2012. Aceptado para publicación: 12 de abril, 2013] 